\title{
A solution of nonlinear Schrödinger equation on metric graphs
}

\author{
K. K. Sabirov ${ }^{1}$, A. R. Khalmukhamedov ${ }^{2}$ \\ ${ }^{1}$ National University of Uzbekistan, 4 University St., 100074, Tashkent, Uzbekistan \\ ${ }^{2}$ Tashkent branch of Moscow State University named after L. V. Lomonosov, \\ 22 Amir Temur St., 100060, Tashkent, Uzbekistan \\ karimjonsabirov@yahoo.com
}

PACS 02.30.Jr,02.30.Ik

DOI 10.17586/2220-8054-2015-6-2-162-172

We treat the Nonlinear Schrödinger equation (NLSE) on Metric graph. An approach developed earlier for NLSE on interval [14], is extended for star graph. Dirichlet boundary conditions are imposed at the ends of bonds are imposed, while continuity conditions are chosen at the vertex of graph.

Keywords: metric graphs, nonlinear Schrödinger equation, solitons.

Received: 2 February 2015

\section{Introduction}

Wave transport in the nonlinear regime, described by nonlinear evolution equations, such as the nonlinear Schrödinger, Korteweg de Vries and sine Gordon equations, has attracted much attention in different areas of physics over the past five decades (see, e.g. books [1] - [5]).

Recently, one can observe a growing interest in particle and wave transport in branched, network type structures [6] - [13]. Such problem is of importance for different topics in physics, such as hydrodynamics, acoustics, optics, cold atom physics and condensed matter physics. Soliton solutions and connection formulae were derived for simplest graphs in the Ref. [6]. The problem of fast solitons on star graphs was treated in the Ref. [7], where the estimates for the transmission and reflection coefficients were obtained in the limit of very high velocities. The problem of soliton transmission and reflection was studied in [9] by numerically solving the stationary NLSE on graphs. The dispersion relations for linear and nonlinear Schrödinger equations on graphs were discussed in [10]. Ref. [11] treated the stationary NLSE in the context of scattering from nonlinear networks. The stationary NLSE with power focusing nonlinearity on star graphs was studied in recent papers $[7,8]$, where the existence of nonlinear stationary states were shown for $\delta$-type boundary conditions. In [13], the exact analytical solutions of the stationary NLSE for simplest graphs were obtained.

In this work, we treat time dependent NLSE on metric graphs by considering the simplest topology, a star graph. Unlike the case of the NLSE on an interval, in the case of the graph, the NLSE becomes a multicomponent equation, with the components related to each other through the boundary conditions given at the graph vertex.

Our aim is to solve the (cubic) nonlinear Schrödinger equation on metric graphs. The latter are systems consisting of bonds which are connected at the vertices [16] to a 
rule which is called the topology of a graph. The topology of a graph can be relayed in terms of a so-called adjacency matrix, which can be written as [17]:

$$
C_{i j}=C_{j i}= \begin{cases}1, & \text { if } i \text { and } j \text { are connected; } \quad i, j=1,2, \ldots, V . \\ 0, & \text { otherwise, }\end{cases}
$$

In the following, we consider the so-called primary star graph, consisting of three bonds connected at single vertex. However, our results can be extended to any arbitrary topology. Our approach is based on extending the method proposed by Fokas and its solution for the NLSE on a finite interval [14]. In Refs. [14,15], an effective method allowing one to obtain a general solution for the NLSE on a finite interval [14] and on a half-line [15] was developed. As it will be shown below, this method can be adapted to the case of the NLSE on a metric graph. Thus, the problem we are going to solve is the nonlinear Schrödinger equation on a graph with bonds $b_{j} \sim\left(0, L_{j}\right), j=1,2,3$, which can be written as:

$$
\begin{array}{r}
i \frac{\partial}{\partial t} q_{j}+\frac{\partial^{2}}{\partial x_{j}^{2}} q_{j}-2 \lambda\left|q_{j}\right|^{2} q_{j}=0, \\
\lambda= \pm 1, \quad j=1,2,3, \quad L_{1}<x_{1}<0,0<x_{2,3}<L_{2,3}, \quad 0<t<T .
\end{array}
$$

The initial conditions are given as:

$$
q_{j}\left(x_{j}, 0\right)=q_{0 j}\left(x_{j}\right), \quad j=1,2,3, \quad L_{1}<x_{1}<0, \quad 0<x_{2,3}<L_{2,3},
$$

The following boundary conditions provide matching of the bonds at the vertex:

$$
\begin{gathered}
q_{1}(0, t)=q_{2}(0, t)=q_{3}(0, t)=g_{0}(t), \quad 0<t<T \\
q_{j}\left(L_{j}, t\right)=f_{0 j}(t), \quad 0<t<T, \quad j=1,2,3, \\
\frac{\partial}{\partial x_{1}} q_{1}(0, t)=\frac{\partial}{\partial x_{2}} q_{2}(0, t)+\frac{\partial}{\partial x_{3}} q_{3}(0, t), \quad 0<t<T
\end{gathered}
$$

Furthermore, we define the following functions:

$$
g_{1 j}(t)=\frac{\partial}{\partial x_{j}} q_{j}(0, t), \quad g_{1 j}(t)=\frac{\partial}{\partial x_{j}} q_{j}\left(L_{j}, t\right) .
$$

These functions are considered to be unknown and will be found subsequently.

The difference between Eq. (1) and with that treated in Ref. [14] is caused by bond indices, $j$. In other words, Eq. (1) is a multicomponent equation in which each component is related to others through the boundary conditions (3) - (5). As we will see below, this makes it possible to rewrite most of the results derived in [14] for the case of metric graphs.

\section{Description of the approach}

The method we are going to utilize includes three steps [14]. The first step consists of Riemann-Hilbert (RH) problem formulation under the assumption of existence. Following Ref. [14], we assume that there exists a smooth solution $q(x, t)=\left\{q_{1}\left(x_{1}, t\right), q_{2}\left(x_{2}, t\right)\right.$, $\left.q_{3}\left(x_{3}, t\right)\right\}$.

Applying the spectral analysis to the Lax pair, we write $q(x, t)=\left\{q_{1}\left(x_{1}, t\right), q_{2}\left(x_{2}, t\right)\right.$, $\left.q_{3}\left(x_{3}, t\right)\right\}$ in terms of the solution of a $2 \times 2$-matrix $\mathrm{RH}$ problem defined in the complex $\mathrm{k}$-plane [14]. Such a problem is uniquely defined in terms of the spectral functions which are given as:

$$
\left\{a_{j}(k), b_{j}(k)\right\}, \quad\left\{A_{j}(k), B_{j}(k)\right\}, \quad\left\{\mathbf{A}_{j}(k), \mathbf{B}_{j}(k)\right\} .
$$


These functions are defined in terms of the functions:

$$
q_{0 j}\left(x_{j}\right), \quad\left\{g_{0}(t), g_{1 j}(t)\right\}, \quad\left\{f_{0 j}(t), f_{1 j}(t)\right\},
$$

respectively. Here, the functions $g_{0}\left(t, g_{1 j}(t)\right.$ and $f_{1 j}(t)$ denote the unknown boundary values for the solution to the NLSE and its derivatives (see Eqs. (3)).

Following Ref. [14], one can show that the spectral functions (6) are not independent, but they satisfy the global relation:

$$
\left(a_{j} \mathbf{A}_{j}+\lambda \bar{b}_{j} e^{2 i k L_{j}} \mathbf{B}_{j}\right) B_{j}-\left(b_{j} \mathbf{A}_{j}+\bar{a}_{j} e^{2 i k L_{j}} \mathbf{B}_{j}\right) A_{j}=e^{4 i k^{2} T} c_{j}^{+}(k), \quad k \in \mathbf{C}_{j},
$$

where $c_{j}^{+}(k)$ has the same meaning as in [14].

The second step implies proof of the existence of the solution to the NLSE, assuming that the above spectral functions satisfy the global relation. The spectral functions given in (6) can be written in terms of the (smooth) functions (7). We also define $q(x, t)=\left\{q_{1}\left(x_{1}, t\right), q_{2}\left(x_{2}, t\right), q_{3}\left(x_{3}, t\right)\right\}$ in terms of the solution of the RH problem formulated in Step 1. Assuming that smooth functions $g_{1 j}(t)$ and $f_{1 j}(t)$ exist such that the spectral functions (6) satisfy the global relation (8), one can prove that:

(i) $q(x, t)=\left\{q_{1}\left(x_{1}, t\right), q_{2}\left(x_{2}, t\right), q_{3}\left(x_{3}, t\right)\right\}$ is defined globally for all $L_{1}<x_{1}<0,0<x_{2,3}<L_{2,3}$, $0<t<T$.

(ii) $q(x, t)=\left\{q_{1}\left(x_{1}, t\right), q_{2}\left(x_{2}, t\right), q_{3}\left(x_{3}, t\right)\right\}$ solves the NLSE.

(iii) $q(x, t)=\left\{q_{1}\left(x_{1}, t\right), q_{2}\left(x_{2}, t\right), q_{3}\left(x_{3}, t\right)\right\}$ satisfies the given initial and boundary conditions:

$$
q_{j}\left(x_{j}, 0\right)=q_{0 j}(x), \quad q_{j}(0, t)=g_{0}(t), \quad q_{j}\left(L_{j}, t\right)=f_{0 j}(t) .
$$

A byproduct of this proof is that:

$$
\frac{\partial}{\partial x_{j}} q_{j}(0, t)=g_{1 j}(t) \text { and } \quad \frac{\partial}{\partial x_{j}} q_{j}\left(L_{j}, t\right)=f_{1 j}(t) .
$$

Finally, the third step presents an analysis of the global relation treated in the second step. Namely, for given $q_{0 j}, g_{0}, f_{0 j}$, one can show that the global relation (8) characterizes $g_{1 j}$ and $f_{1 j}$ through the solution of a system of nonlinear Volterra integral equations.

Furthermore, following the Ref. [14], we introduce the eigenfunctions, $\left\{\mu_{j}^{(n)}(x, t, k)\right\}_{n=1}^{4}$, such that:

$$
\begin{array}{r}
\mu_{j}^{(1)}(0, T, k)=I, \quad \mu_{j}^{(2)}(0,0, k)=I, \\
\mu_{j}^{(3)}\left(L_{j}, 0, k\right)=I, \quad \mu_{j}^{(4)}\left(L_{j}, T, k\right)=I, \quad j=1,2,3,
\end{array}
$$

with $\mu_{j}^{(n)}$ being the $2 \times 2$ matrices, $I=\operatorname{diag}(1,1)$. One can show that these eigenfunctions can be written in terms of the matrices $s_{j}, S_{j}, S_{j}^{L}$ as:

$$
\begin{aligned}
s_{j}(k)=\mu_{j}^{(3)}(0,0, k), \quad S_{j}(k) & =\left(e^{2 i k^{2} T \sigma_{3}} \mu_{j}^{(2)}(0, T, k) e^{-2 i k^{2} T \sigma_{3}}\right)^{-1} \\
S_{j}^{L}(k) & =\left(e^{2 i k^{2} T \sigma_{3}} \mu_{j}^{(3)}\left(L_{j}, T, k\right) e^{-2 i k^{2} T \sigma_{3}}\right)^{-1}
\end{aligned}
$$

where $\sigma_{3}=\operatorname{diag}(1,-1)$. 


\section{Lax pair and its solutions}

The Lax pair for our problem can be written as [20]:

$$
\frac{\partial}{\partial x} \mu_{j}+i k \hat{\sigma}_{3, j} \mu_{j}=Q_{j} \mu_{j}, \quad \frac{\partial}{\partial t} \mu_{j}+2 i k^{2} \hat{\sigma}_{3, j} \mu_{j}=\tilde{Q}_{j} \mu_{j}
$$

where $\mu_{j}(x, t, k)$ is a $2 \times 2$ matrix-valued function, $\hat{\sigma}_{3, j}$ is defined by:

$$
\hat{\sigma}_{3, j}=\left[\sigma_{3, j}, \cdot\right] \quad \sigma_{3, j}=\operatorname{diag}(1,-1)
$$

and the $2 \times 2$ matrices $Q_{j}, \tilde{Q}_{j}$ are given as:

$$
\begin{array}{r}
Q_{j}\left(x_{j}, t\right)=\left(\begin{array}{cc}
0 & q_{j}\left(x_{j}, t\right) \\
\lambda \bar{q}_{j}\left(x_{j}, t\right) & 0
\end{array}\right), \\
\tilde{Q}_{j}\left(x_{j}, t, k\right)=2 k Q_{j}-i \frac{\partial}{\partial x} Q_{j} \sigma_{3, j}-i \lambda\left|q_{j}\right|^{2} \sigma_{3, j}, \quad \lambda= \pm 1 .
\end{array}
$$

Furthermore, we assume that there exists a sufficiently smooth solution $q_{j}\left(x_{j}, t\right)$, $j=1,2,3, x_{1} \in\left[L_{1}, 0\right], x_{2,3} \in\left[0, L_{2,3}\right], t \in[0, T]$, of NLSE.

A solution of equation (11) is given by [14]:

$$
\mu_{j}^{(*)}\left(x_{j}, t, k\right)=I+\int_{\left(x_{j *}, t_{*}\right)}^{\left(x_{j}, t\right)} e^{-i\left(k x_{j}+2 k^{2} t\right) \hat{\sigma}_{3, j}} W_{j}(y, \tau, k),
$$

where the closed 1 -form $W_{j}$ is defined by:

$$
W_{j}=e^{i\left(k x+2 k^{2} t\right) \hat{\sigma}_{3, j}}\left(Q_{j} \mu_{j} d x+\tilde{Q}_{j} \mu_{j} d t\right),
$$

$\left(x_{j *}, t_{*}\right)$ is an arbitrary point in the domain $x_{1} \in\left[L_{1}, 0\right], x_{2,3} \in\left[0, L_{2,3}\right], t \in[0, T]$, and the integral denotes a line integral connecting smoothly the points indicated. equations:

Following Ref. [14], it can be shown that the functions $\mu_{j}^{(n)}$ are related by these

$$
\begin{aligned}
& \mu_{j}^{(3)}\left(x_{j}, t, k\right)=\mu_{j}^{(2)}\left(x_{j}, t, k\right) e^{-i\left(k x_{j}+2 k^{2} t\right) \hat{\sigma}_{3, j}} s_{j}(k), \\
& \mu_{j}^{(1)}\left(x_{j}, t, k\right)=\mu_{j}^{(2)}\left(x_{j}, t, k\right) e^{-i\left(k x_{j}+2 k^{2} t\right) \hat{\sigma}_{3, j}} S_{j}(k), \\
& \mu_{j}^{(4)}\left(x_{j}, t, k\right)=\mu_{j}^{(3)}\left(x_{j}, t, k\right) e^{-i\left(k x_{j}+2 k^{2} t\right) \hat{\sigma}_{3, j}} S_{j}^{L}(k),
\end{aligned}
$$

and one can find from Eq. (16) at $x_{j}=t=0, s(k)=\mu_{j}^{(3)}(0,0, k)$. Finally, from Eqs. (17) and (18) at $x_{j}=L_{j}, t=T$ we have:

$$
S_{j}^{L}(k)=\left(e^{2 i k^{2} T \hat{\sigma}_{3, j}} \mu_{j}^{(3)}\left(L_{j}, T, k\right)\right)^{-1}
$$

and

$$
\mu_{j}^{(4)}\left(x_{j}, t, k\right)=\mu_{j}^{(2)}\left(x_{j}, t, k\right) e^{-i\left(k x_{j}+2 k^{2} t\right) \hat{\sigma}_{3, j}}\left(s(k) e^{i k L_{j} \hat{\sigma}_{3, j}} S_{j}^{L}(k)\right) .
$$




\section{The global relation}

As was mentioned before, the spectral functions $a_{j}(k), b_{j}(k), A_{j}(k), B_{j}(k), \mathbf{A}_{j}(k)$, $\mathbf{B}_{j}(k)$ are not independent, but they satisfy the global relation (8), where $c_{j}^{+}(k)$ denotes the element of $-\int_{0}^{L_{j}}\left[\exp \left(i k y \hat{\sigma}_{3, j}\right)\right]\left(Q_{j} \mu_{j}^{(4)}\right)(y, T, k) d y$, and $\mu_{j}^{(4)}$ is defined by an equation similar to $\mu_{j}^{(3)}$ with $\int_{0}^{t}$ replaced by $-\int_{0}^{T}$. The proof is the same as in the case of the NLSE for the finite interval treated in [14]. We now introduce $M_{j}\left(x_{j}, t, k\right)$, defined by:

$$
\begin{gathered}
M_{j}^{(+)}=\left(\frac{\mu_{j}^{(2)(1)}}{\alpha_{j}(k)}, \mu_{j}^{(4)(1)}\right), \quad \arg k \in\left[0, \frac{\pi}{2}\right], \\
M_{j}^{(-)}=\left(\frac{\mu_{j}^{(1)(2)}}{d_{j}(k)}, \mu_{j}^{(3)(2)}\right), \quad \arg k \in\left[\frac{\pi}{2}, \pi\right], \\
M_{j}^{(+)}=\left(\mu_{j}^{(3)(3)}, \frac{\mu_{j}^{(1)(3)}}{\overline{d_{j}(\bar{k})}}\right), \quad \arg k \in\left[\pi, \frac{3 \pi}{2}\right], \\
M_{j}^{(-)}=\left(\mu_{j}^{(4)(4)}, \frac{\mu_{j}^{(2)(4)}}{\overline{\alpha_{j}(\bar{k})}}\right), \quad \arg k \in\left[\frac{3 \pi}{2}, 2 \pi\right],
\end{gathered}
$$

where the scalars $d_{j}(k)$ and $\alpha_{j}(k)$ are defined below. These definitions imply:

$$
\operatorname{det} M_{j}\left(x_{j}, t, k\right)=1
$$

and

$$
M_{j}\left(x_{j}, t, k\right)=I+O\left(\frac{1}{k}\right), \quad k \rightarrow \infty
$$

As in the case of the NLSE on a finite graph studied in [14], it can be shown that $M_{j}$ satisfies the jump condition:

$$
M_{j}^{(-)}\left(x_{j}, t, k\right)=M_{j}^{(+)}\left(x_{j}, t, k\right) J_{j}\left(x_{j}, t, k\right), \quad k \in \mathbf{R} \cup i \mathbf{R},
$$

where the $2 \times 2$ matrix $J_{j}$ is defined by:

$$
J_{j}=\left[\begin{array}{cl}
J_{j}^{(2)}, & \arg k=0 ; \\
J_{j}(1), & \arg k=\frac{\pi}{2} ; \\
J_{j}^{(4)} \equiv J_{j}^{(3)}\left(J_{j}^{(2)}\right)^{-1} J_{j}^{(1)}, & \arg k=\pi ; \\
J_{j}^{(3)}, & \arg k=\frac{3 \pi}{2}
\end{array}\right.
$$


and

$$
\begin{aligned}
& J_{j}^{(1)}=\left(\begin{array}{cc}
\frac{\delta_{j}(k)}{d_{j}(k)} & -\mathbf{B}_{j}(k) e^{2 i k L_{j}} e^{-2 i \theta_{j}} \\
\frac{\lambda B_{j}(\bar{k})}{d_{j}(k) \alpha_{j}(k)} e^{2 i \theta_{j}} & \frac{a_{j}(k)}{\alpha_{j}(k)}
\end{array}\right), \\
& J_{j}^{(3)}=\left(\begin{array}{cc}
\overline{\frac{\delta_{j}(\bar{k})}{\overline{d(\bar{k})}}} & \overline{\frac{-B_{j}(k)}{d_{j}(\bar{k}) \alpha_{j}(\bar{k})}} e^{-2 i \theta_{j}} \\
\lambda \overline{a(\bar{k})} \\
\overline{\mathbf{B}_{j}(\bar{k})} e^{-2 i k L_{j}} e^{2 i \theta_{j}} & \frac{\alpha_{j}(\bar{k})}{\beta_{j}(k)}
\end{array}\right), \\
& J_{j}^{(2)}=\left(\begin{array}{cc}
1 & -\frac{\beta_{j}(k)}{\overline{\alpha_{j}(k)}} e^{-2 i \theta_{j}} \\
\lambda \frac{1}{\beta_{j}(k)} e^{2 i \theta_{j}} & \frac{1}{\left|\alpha_{j}(k)\right|^{2}}
\end{array}\right), \\
& \theta_{j}\left(x_{j}, t, k\right)=k x_{j}+2 k^{2} t, \\
& \alpha_{j}(k)=a_{j}(k) \mathbf{A}_{j}(k)+\lambda \overline{b_{j}(\bar{k})} e^{2 i k L_{j}} \mathbf{B}_{j}(k), \\
& \beta_{j}(k)=b_{j}(k) \mathbf{A}_{j}(k)+\lambda \overline{a_{j}(\bar{k})} e^{2 i k L_{j}} \mathbf{B}_{j}(k) \\
& d_{j}(k)=a_{j}(k) \overline{\mathbf{A}_{j}(\bar{k})}-\lambda b_{j}(k) e^{2 i k L_{j}} \overline{\mathbf{B}_{j}(\bar{k})}, \\
& \delta_{j}(k)=\alpha_{j}(k) \overline{\mathbf{A}_{j}(\bar{k})}-\lambda \beta_{j}(k) e^{2 i k L_{j}} \overline{\mathbf{B}_{j}(\bar{k})} .
\end{aligned}
$$

The above expressions are the same as those for the NLSE on a finite interval, except for the bond index, $j$.

Following Ref. [14], one can prove

Theorem. Let $q_{0 j}(x)$ be a smooth function. We assume that that the set of functions $g_{0}(t)$, $g_{1 j}(t), f_{0 j}(t), f_{1 j}(t)$, is admissible with respect to $q_{0 j}(x)$ and define the spectral functions $a_{j}(k), b_{j}(k), A_{j}(k), B_{j}(k), \mathbf{A}_{j}(k), \mathbf{B}_{j}(k)$ in terms of $q_{0 j}(x), g_{0}(t), g_{1 j}(t), f_{0 j}(t), f_{1 j}(t)$. We assume that

- $a_{j}(k)$ has at most simple zeros, $\left\{k_{j}^{(n)}\right\}$, for $\Im k_{j}^{(n)}>0$ and has no zeros for $\Im k=0$.

- $A_{j}(k)$ has at most simple zeros, $\left\{K_{j}^{(n)}\right\}$, for $\arg K_{j}^{(n)} \in\left(0, \frac{\pi}{2}\right) \cup\left(\pi, \frac{3 \pi}{2}\right)$ and has no zeros for $\arg k=0, \frac{\pi}{2}, \pi, \frac{3 \pi}{2}$.

- $\mathbf{A}_{j}(k)$ has at most simple zeros, $\left\{\mathbf{K}_{j}^{(n)}\right\}$, for $\arg \mathbf{K}_{j}^{(n)} \in\left(0, \frac{\pi}{2}\right) \cup\left(\pi, \frac{3 \pi}{2}\right)$ and has no zeros for $\arg k=0, \frac{\pi}{2}, \pi, \frac{3 \pi}{2}$.

- The function

$$
d_{j}(k)=a_{j}(k) \overline{A_{j}(\bar{k})}-\lambda b_{j}(k) \overline{B_{j}(\bar{k})}
$$

has at most simple zeros, $\left\{\lambda_{j}^{(n)}\right\}$, for $\arg \lambda_{j}^{(n)} \in\left(\frac{\pi}{2}, \pi\right)$ and has no zeros for $\arg k=\frac{\pi}{2}$ and $\arg k=\pi$.

- The function

$$
\alpha_{j}(k)=a_{j}(k) \mathbf{A}_{j}(k)+\lambda \overline{b(\bar{k})} e^{2 i k L_{j}} \mathbf{B}_{j}(k)
$$


has at most simple zeros, $\left\{v_{j}^{(n)}\right\}$, for $\arg v_{j}^{(n)} \in\left(0, \frac{\pi}{2}\right)$ and has no zeros for $\arg k=0$, $\arg k=\frac{\pi}{2}$.

- None of the zeros of $a_{j}(k)$ for $\arg k \in\left(\frac{\pi}{2}, \pi\right)$ coincides with a zero of $d_{j}(k)$.

- None of the zeros of $a_{j}(k)$ for $\arg k \in\left(0, \frac{\pi}{2}\right)$ coincides with a zero of $\alpha_{j}(k)$.

- None of the zeros of $\alpha_{j}(k)$ for $\arg k \in\left(0, \frac{\pi}{2}\right)$ coincides with a zero of $A_{j}(k)$ or a zero of $\mathbf{A}_{j}(k)$.

- None of the zeros of $d_{j}(k)$ for $\arg k \in\left(\frac{\pi}{2}, \pi\right)$ coincides with a zero of $\overline{A_{j}(\bar{k})}$ or a zero of $\overline{\mathbf{A}_{j}(\bar{k})}$.

We define $M_{j}\left(x_{j}, t, k\right)$ as the solution of the following $2 \times 2$ matrix RH problem:

- $M_{j}$ is sectionally meromorphic in $\mathbf{C} /\{\mathbf{R} \cup \mathrm{i} \mathbf{R}\}$, and has unit determinant.

$$
M_{j}^{(-)}\left(x_{j}, t, k\right)=M_{j}^{(+)}\left(x_{j}, t, k\right) J_{j}\left(x_{j}, t, k\right), \quad k \in \mathbf{R} \cup \mathrm{i} \mathbf{R},
$$

where $M_{j}$ is $M_{j}^{(-)}$for $\arg k \in\left[\frac{\pi}{2}, \pi\right] \cup\left[\frac{3 \pi}{2}, 2 \pi\right], M_{j}$ is $M_{j}^{(+)}$for $\arg k \in\left[0, \frac{\pi}{2}\right] \cup$ $\left[\pi, \frac{3 \pi}{2}\right]$, and $J_{j}$ is defined in terms of $a_{j}, b_{j}, A_{j}, B_{j}, \mathbf{A}_{j}, \mathbf{B}_{j}$, by equations (24) and (25).

$$
M_{j}\left(x_{j}, t, k\right)=I+O\left(\frac{1}{k}\right), \quad k \rightarrow \infty .
$$

- Let $\left[M_{j}\right]_{1}$ and $\left[M_{j}\right]_{2}$ denote the first and the second column of the matrix $M_{j}$. Then residue conditions:

$$
\begin{gathered}
\operatorname{Res}_{k=v_{j}^{(n)}}\left[M_{j}\left(x_{j}, t, k\right)\right]_{1}=c_{j}^{(n)(1)} e^{4 i\left(v_{j}^{(n)}\right)^{2} t+2 i v_{j}^{(n)} x_{j}}\left[M_{j}\left(x_{j}, t, v_{j}^{(n)}\right)\right]_{2}, \\
\underset{k=\overline{v_{j}^{(n)}}}{\operatorname{Res}}\left[M_{j}\left(x_{j}, t, k\right)\right]_{2}=\lambda \overline{c_{j}^{(n)(1)}} e^{-4 i\left(v_{j}^{(n)}\right)^{2} t-2 i v_{j}^{(n)} x_{j}}\left[M_{j}\left(x_{j}, t, \bar{v}_{j}^{(n)}\right)\right]_{1}, \\
\quad \operatorname{Res}_{k=\lambda_{j}^{(n)}}\left[M_{j}\left(x_{j}, t, k\right)\right]_{1}=c_{j}^{(n)(2)} e^{4 i\left(v_{j}^{(n)}\right)^{2} t+2 i v_{j}^{(n)} x_{j}}\left[M_{j}\left(x_{j}, t, \lambda_{j}^{(n)}\right)\right]_{2}, \\
\underset{k=\overline{\lambda_{j}^{(n)}}}{\operatorname{Res}_{j}}\left[M_{j}\left(x_{j}, t, k\right)\right]_{2}=\lambda \overline{c_{j}^{(n)(2)}} e^{-4 i\left(v_{j}^{(n)}\right)^{2} t-2 i v_{j}^{(n)} x_{j}}\left[M_{j}\left(x_{j}, t, \bar{\lambda}_{j}^{(n)}\right)\right]_{1},
\end{gathered}
$$

where:

$$
c_{j}^{(n)(1)}=\frac{a_{j}\left(v_{j}^{(n)}\right)}{e^{2 i v_{j}^{(n)} L_{j}} \mathbf{B}_{j}\left(v_{j}^{(n)}\right) \dot{\alpha}_{j}\left(v_{j}^{(n)}\right)}, \quad c_{j}^{(n)(2)}=\frac{\lambda \overline{B_{j}\left(\bar{\lambda}_{j}^{(n)}\right)}}{a_{j}\left(\lambda_{j}^{(n)}\right) \dot{d}_{j}\left(\lambda_{j}^{(n)}\right)} .
$$

by

Then, $M_{j}\left(x_{j}, t, k\right)$ exists and is unique. We define $q_{j}\left(x_{j}, t\right)$ in terms of $M_{j}\left(x_{j}, t, k\right)$

$$
q_{j}(x, t)=2 \mathrm{i} \cdot \lim _{k \rightarrow \infty} k\left(M_{j}(x, t, k)\right)_{12} .
$$

Then, $q_{j}(x, t)$, together with the following functions: 


$$
\begin{gathered}
q_{j}(x, 0)=q_{0 j}(x), \quad q_{j}(0, t)=g_{0}(t), \quad \frac{\partial}{\partial x} q_{j}(0, t)=g_{1 j}(t), \\
q_{j}\left(L_{j}, t\right)=f_{0 j}(t), \quad \frac{\partial}{\partial x} q_{j}\left(L_{j}, t\right)=f_{1 j}(t)
\end{gathered}
$$

present the solution for the nonlinear Schrödinger equation (1) with initial and boundary conditions given by Eqs.(2) - (5), respectively. The proof of the theorem is similar to that of the NLSE for a finite interval treated in [14]).

Furthermore, repeating the same steps as in [14] ( for $a_{j}(k) \equiv 1, b_{j}(k) \equiv 0$ ), we get the following expressions for $f_{1 j}, g_{1 j}$ :

$$
\begin{array}{r}
\frac{i \pi}{4} f_{1 j}=\int_{\partial D_{1}^{0}} \frac{2 k^{2}}{\Delta_{j}(k)}\left[\hat{M}_{j}^{(1)}(t, k)-\frac{g_{0}(t)}{2 i k^{2}}\right] d k-\int_{\partial D_{1}^{0}} k^{2} \frac{\Sigma_{j}(k)}{\Delta_{j}(k)}\left[\hat{\mathbf{M}}_{j}^{(1)}(t, k)-\frac{f_{0 j}(t)}{2 i k^{2}}\right] d k+ \\
\quad+\int_{\partial D_{1}^{0}} \frac{k}{\Delta_{j}(k)}\left[F_{j}(t, k)-F_{j}(t,-k)\right] d k, \\
-\frac{i \pi}{4} g_{1 j}=\int_{\partial D_{1}^{0}} \frac{2 k^{2}}{\Delta_{j}(k)}\left[\hat{\mathbf{M}}_{j}^{(1)}(t, k)-\frac{f_{0 j}(t)}{2 i k^{2}}\right] d k-\int_{\partial D_{1}^{0}} k^{2} \frac{\Sigma_{j}(k)}{\Delta_{j}(k)}\left[\hat{M}_{j}^{(1)}(t, k)-\frac{g_{0}(t)}{2 i k^{2}}\right] d k- \\
-\int \frac{k}{\Delta_{j}(k)}\left[e^{-2 i k L_{j}} F_{j}(t, k)-e^{2 i k L_{j}} F_{j}(t,-k)\right] d k,
\end{array}
$$

where:

$$
\begin{aligned}
& \Sigma_{j}(k)=e^{2 i k L_{j}}+e^{-2 i k L_{j}}, \\
& F_{j}(t, k)=\frac{i f_{0 j}(t)}{2} e^{2 i k L_{j}} \hat{\mathbf{M}}_{j}^{(2)}-\frac{i g_{0}(t)}{2} \hat{M}_{j}^{(2)}+ \\
& +\left[\overline{\hat{\mathbf{L}}_{j}^{(2)}}-i \lambda \frac{f_{0 j}(t)}{2} \overline{\hat{\mathbf{M}}_{j}^{(1)}}+k \overline{\hat{\mathbf{M}}_{j}^{(2)}}\right] \cdot\left[\hat{L}_{j}^{(1)}-i \frac{g_{0}(t)}{2} \hat{M}_{j}^{(2)}+k \hat{M}_{j}^{(1)}\right]- \\
& -e^{2 i k L_{j}}\left[\overline{\hat{L}_{j}^{(2)}}-i \lambda \frac{g_{0}(t)}{2} \overline{\hat{M}_{j}^{(1)}}+k \overline{\hat{M}_{j}^{(2)}}\right] \cdot\left[\hat{\mathbf{L}}_{j}^{(1)}-i \frac{f_{0 j}(t)}{2} \hat{\mathbf{M}}_{j}^{(2)}+k \hat{\mathbf{M}}_{j}^{(1)}\right]
\end{aligned}
$$

Finally, from Eqs. (40) and (5) we obtain: 


$$
\begin{aligned}
& g_{0}(t)=\frac{\int_{\partial D_{1}^{0}} k^{2} \frac{\Sigma_{1}(k)}{\Delta_{1}(k)} \hat{M}_{1}^{(1)} d k-\int_{\partial D_{1}^{0}} \frac{2 k^{2}}{\Delta_{1}(k)}\left[\hat{\mathbf{M}}_{1}^{(1)}-\frac{f_{01}(t)}{2 i k^{2}}\right] d k}{\frac{1}{2 i} \int_{\partial D_{1}^{0}} \frac{\Sigma_{1}(k)}{\Delta_{1}(k)} d k-\frac{1}{2 i} \sum_{j=2}^{3} \int_{\partial D_{1}^{0}} \frac{\Sigma_{j}(k)}{\Delta_{j}(k)} d k}- \\
& -\frac{\sum_{j=2}^{3}\left\{\int_{\partial D_{1}^{0}} k^{2} \frac{\Sigma_{j}(k)}{\Delta_{j}(k)} \hat{M}_{j}^{(1)} d k-\int_{\partial D_{1}^{0}} \frac{2 k^{2}}{\Delta_{j}(k)}\left[\hat{\mathbf{M}}_{j}^{(1)}-\frac{f_{0 j}(t)}{2 i k^{2}}\right] d k\right\}}{\frac{1}{2 i} \int_{\partial D_{1}^{0}} \frac{\Sigma_{1}(k)}{\Delta_{1}(k)} d k-\frac{1}{2 i} \sum_{j=2}^{3} \int_{\partial D_{1}^{0}} \frac{\Sigma_{j}(k)}{\Delta_{j}(k)} d k}+ \\
& +\frac{\int_{\partial D_{1}^{0}} \frac{k}{\Delta_{1}(k)}\left[e^{-2 i k L_{1}} F_{1}(t, k)-e^{2 i k L_{1}} F_{1}(t,-k)\right] d k}{\frac{1}{2 i} \int_{\partial D_{1}^{0}} \frac{\Sigma_{1}(k)}{\Delta_{1}(k)} d k-\frac{1}{2 i} \sum_{j=2}^{3} \int_{\partial D_{1}^{0}} \frac{\Sigma_{j}(k)}{\Delta_{j}(k)} d k}- \\
& -\frac{\sum_{j=2}^{3} \int_{\partial D_{1}^{0}} \frac{k}{\Delta_{j}(k)}\left[e^{-2 i k L_{j}} F_{j}(t, k)-e^{2 i k L_{j}} F_{j}(t,-k)\right] d k}{\frac{1}{2 i} \int_{\partial D_{1}^{0}} \frac{\Sigma_{1}(k)}{\Delta_{1}(k)} d k-\frac{1}{2 i} \sum_{j=2}^{3} \int_{\partial D_{1}^{0}} \frac{\Sigma_{j}(k)}{\Delta_{j}(k)} d k} .
\end{aligned}
$$

We note that $\mu_{j}^{(1)}(0, t, k)$ and $\mu_{j}^{(2)}(0, t, k)$ are solutions of:

$$
\frac{\partial}{\partial t} \mu_{j}+2 i k^{2} \hat{\sigma}_{3, j} \mu_{j}=\tilde{Q}_{j}(0, t, k) \mu_{j},
$$

where:

$$
\tilde{Q}_{j}(0, t, k)=\left(\begin{array}{cc}
-i \lambda\left|q_{j}(0, t)\right|^{2} & 2 k q_{j}(0, t)+i \frac{\partial}{\partial x_{j}} q_{j}(0, t) \\
2 k \bar{q}_{j}(0, t)-i \frac{\partial}{\partial x_{j}} \bar{q}_{j}(0, t) & i \lambda\left|q_{j}(0, t)\right|^{2}
\end{array}\right) .
$$

Therefore, it satisfies:

$$
\tilde{Q}_{j}(0, t, k)=\sigma_{3, j} \Sigma \overline{\tilde{Q}_{j}(0, t, \bar{k})} \Sigma \sigma_{3, j},
$$

where $\overline{\tilde{Q}_{j}(0, t, \bar{k})}=\left(\begin{array}{cc}i \lambda\left|q_{j}(0, t)\right|^{2} & 2 k \bar{q}_{j}(0, t)+i \frac{\partial}{\partial x_{j}} \bar{q}_{j}(0, t) \\ 2 k q_{j}(0, t)-i \frac{\partial}{\partial x_{j}} q_{j}(0, t) & -i \lambda\left|q_{j}(0, t)\right|^{2}\end{array}\right), \quad \Sigma=\left(\begin{array}{cc}\lambda & 0 \\ 0 & \lambda\end{array}\right)$.

This implies the following symmetry for boundary scattering matrix:

$$
S_{j}(k)=\sigma_{3, j} \Sigma \overline{S_{j}(\bar{k})} \Sigma \sigma_{3, j},
$$


where $\overline{S_{j}(\bar{k})}=\left(\begin{array}{cc}A_{j}(k) & \overline{B_{j}(\bar{k})} \\ \lambda B_{j}(k) & \overline{A_{j}(\bar{k})}\end{array}\right)$.

\section{Conclusions}

In this paper, we treated the nonlinear Schrödinger equation with cubic nonlinearity on a metric graph. The boundary conditions were imposed to provide continiuty and current conservation at the graph vertex. Our approach is based an extension applied earlier by Fokas [14] for the solution to the NLSE on a finite interval with Dirichlet boundary conditions. Unlike the case of the NLSE on the interval in [14], in the case of our graph, we have:

i) Multicomponent NLSE, whose components are related to each other through the vertex boundary conditions.

ii) Additional, Neumann type boundary conditions at the graph vertex.

iii)Additional unknown functions, $g_{0}, g_{1}, f_{0}, f_{1}$ in the initial and boundary conditions. However, this doesn't lead to serious complication for adopting method of [14] for the case of graphs, although results obtained are completely different from those of NLSE for finite interval. We note that the above treatment of NLSE on star graph can be extended to other graph topologies as well.

\section{References}

[1] Y.S. Kivshar and G.P. Agarwal. Optical Solitons: From Fibers to Photonic Crystals. Academic, San Diego (2003).

[2] M.J. Ablowitz and P.A. Clarkson. Solitons, Nonlinear Evolution Equations and Inverse Scattering. Cambridge: Cambridge University Press (1999).

[3] C.J. Pethick and H. Smith. Bose-Einstein Condensation in Dilute Gases. Cambridge University Press, Cambridge, England (2002).

[4] L. Pitaevskii and S. Stringari. Bose-Einstein Condensation. Oxford University Press, Oxford, England (2003).

[5] T. Dauxois and M. Peyrard. Physics of Solitons. Cambridge University Press, Cambridge, England (2006).

[6] Z. Sobirov, D. Matrasulov, K. Sabirov, et al. Integrable nonlinear Schrodinger equation on simple networks: Connection formula at vertices. Phys. Rev. E, 81, P. 066602 (2010).

[7] R. Adami, C. Cacciapuoti, D. Finco, D. Noja. Fast solitons on star graphs. Reviews in Mathematical Physics, 23 (04), P. 409-451 (2011).

[8] R. Adami, C. Cacciapuoti, D. Finco, D. Noja. On the structure of critical energy levels for the cubic focusing NLS on star graphs. Journal of Physics A: Mathematical and Theoretical, 45 (19), P. 192001 (2012).

[9] R.C. Cascaval and C.T. Hunter. Linear and nonlinear Schrodinger equations on simple networks. Libertas Mathematica, 30, P. 85-98 (2010).

[10] V. Banica and L.I. Ignat. Dispersion for the Schrödinger equation on networks Journal of Mathematical Physics; 52 (8), P. 083703-083703-14 (2011).

[11] S. Gnutzmann, U. Smilansky, and S. Derevyanko. Stationary scattering from a nonlinear network. Phys. Rev. A, 83, P. 033831 (2011).

[12] R. Adami, D. Noja, C. Ortoleva. Orbital and asymptotic stability for standing waves of a nonlinear Schrödinger equation with concentrated nonlinearity in dimension three. Journal of Mathematical Physics, 54, P. 013501-013533 (2013).

[13] K.K. Sabirov, Z.A. Sobirov, D. Babajanov and D.U. Matrasulov. Stationary Nonlinear Schrödinger Equation on Simplest Graphs. Phys.Lett. A, 377, P. 860-865 (2013).

[14] A.S. Fokas and A.R. Its. The nonlinear Schrödinger equation on the interval. J. Phys. A: Math. Gen, 37 P. 6091 (2004).

[15] A.S. Fokas, A.R. Its and L.-Y. Sung. The nonlinear Schrödinger equation on the half-line. Nonlinearity, 18, P. 1771 (2005). 
[16] F. Harary. Graph Theory. Addison-Wesley, Reading (1969), 274 p.

[17] T. Kottos and U. Smilansky. Periodic Orbit Theory and Spectral Statistics for Quantum Graphs. Ann.Phys., 274 (1) P. 76-124 (1999).

[18] A. Boutet de Monvel and V.Kotlyarov. Scattering problem for the Zakharov-Shabat equations on the semi-axis. Inverse Problems, 16, P. 1813 (2000).

[19] P.D. Lax. Integrals of nonlinear equations of evolution and solitary waves. Commun. Pure Appl. Math., 21, P. 467-490 (1968).

[20] V.E. Zakharov and A. Shabat. Exact theory of two-dimensional self-focusing and one-dimentional selfmodulation of waves in nonlinear media. Sov. Phys. JETP, 34 (1), P. 62-69 (1972) 